\title{
En Afrique australe - Moments et enseignements d'un périple
}

\section{Jean Martin}

En octobre 2014, j'ai passé trois semaines dans le Sud de l'Afrique, dans quatre pays (République sud-africaine, Namibie, Botswana et Zimbabwe), du Cap aux Chutes Victoria. L'idée était de découvrir la géographie/géologie de cette région, la nature, des animaux dans les parcs nationaux. J'ai aussi cherché à "sentir» les pays traversés, leurs populations et conditions d'existence, à la lumière de leur histoire complexe, mouvementée, belliqueuse (l'Afrique du Sud a connu aux $\mathrm{XIX}^{\mathrm{e}}$ et $\mathrm{XX}^{\mathrm{e}}$ siècles une assez incroyable succession de guerres, entre adversaires "changeants»).

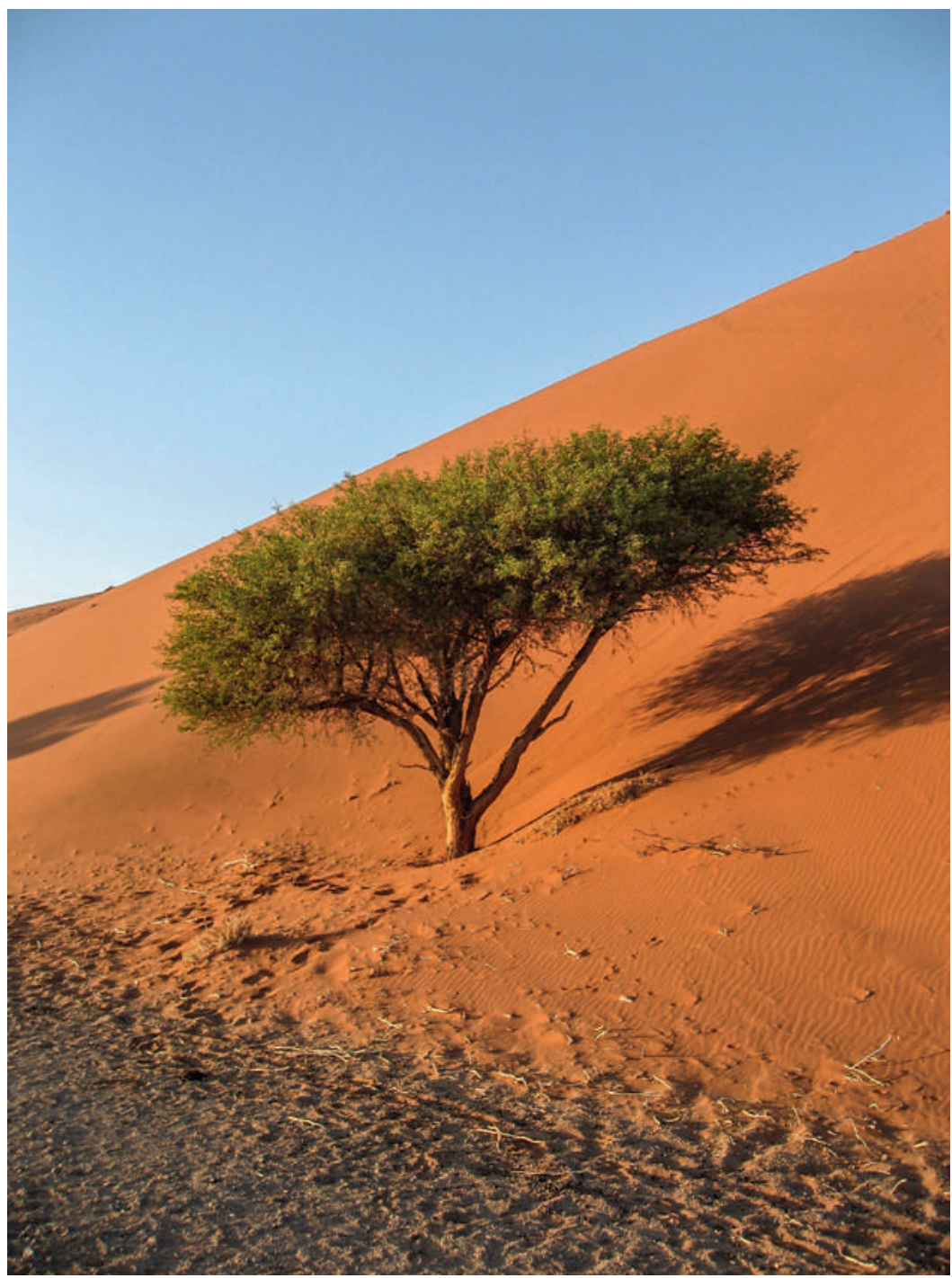

Le désert du Namib, près de Sesriem - certaines dunes atteignent $300 \mathrm{~m}$ de haut.
De la ville du Cap, nous sommes partis vers le Nord, du côté ouest du continent, jusqu'en Namibie: ville de Swakopmund et port de Walvis Bay; désert de sable puis région spectaculaire de beaux massifs montagneux et de parcs nationaux (Namibie puis Botswana), où la faune et aussi la flore sont l'intérêt majeur. Pour finir aux Chutes Victoria, découvertes en 1855 par David Livingstone, le missionnaire explorateur qui a parcouru cette partie de l'Afrique durant 25 ans. Dans les vignettes qui suivent, quelquesunes de mes impressions.

Le Cap. Belle ville, baie magnifique - en concurrence, s'agissant d'être la plus belle du monde et pour celles que j'ai eu la chance de voir, avec San Francisco, Rio, Diego Suarez (Madagascar) ou Halong (Vietnam). Centre historique intéressant, très attrayant "Waterfront" moderne, avec le Robben Island Museum consacré à Nelson Mandela et ses 27 années de prison, dont 18 dans cette île. A noter, collègues touristes potentiels, le "African Trading Post", maison portuaire restaurée, superbe (et plutôt avantageux) marché d'art africain, rempli d'objets qui mériteraient plusieurs musées. Remarquable aquarium «des deux Océans». Tour au Cap de Bonne Espérance, que nous n'avons pas trouvé très spectaculaire.

Ville de Springbok (province de Northern Cape, Afrique du Sud). C'est le Namakwaland, pays d'une des ethnies de Bushmen, de ces indigènes au type physique différent qui parviennent/parvenaient à survivre dans des milieux très arides. Springbok est un «middle of nowhere», qui m’a fasciné comme me fascinent tous les milieux de nulle part ou les finistères/bouts du monde. Pancarte devant un café: «Nous sommes ouverts mais la porte est fermée à cause des conditions météo» (vent). Ambiance de ce que les Américains appellent "frontier», un Far West africain. Avec aussi ceux qui ne sont pas parvenus à s'insérer dans la société autre, nouvelle: paumés, alcooliques, miséreux (y compris un Blanc pauvre - il $\mathrm{y}$ a en a-mendiant à la porte de notre bus).

Townships. Issus de l'apartheid, ils ont été créés par les maîtres blancs de l'époque: quartiers d'habitations standards très modestes où les Noirs étaient tenus de se loger, par groupe ethnique. Des mouvements de résistance s'y sont développés, avec des ré- 


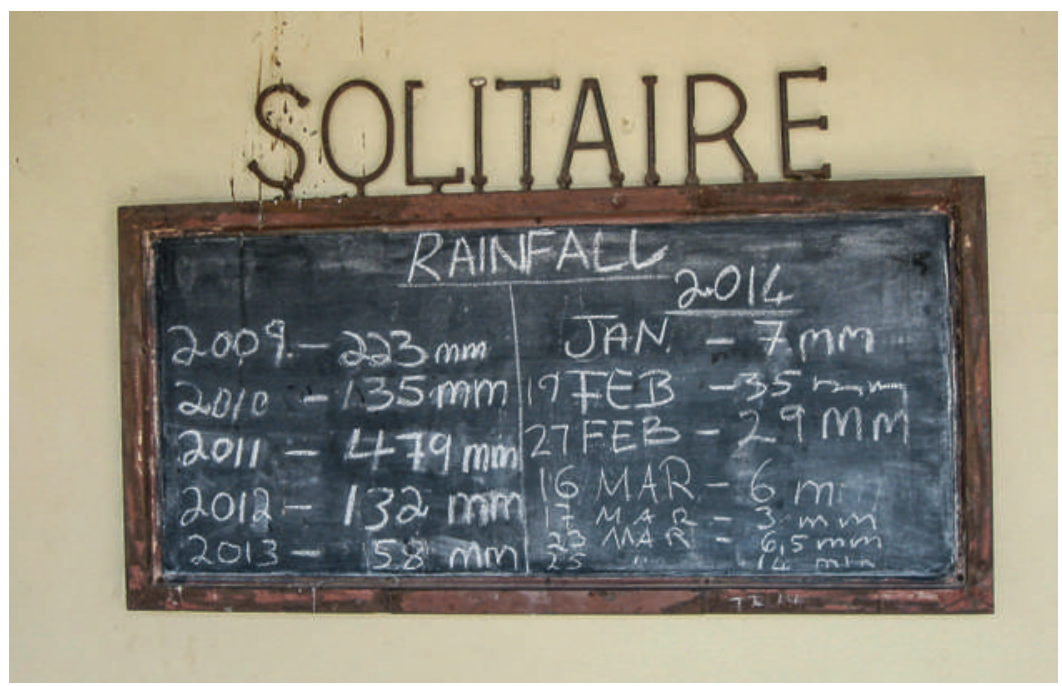

A Solitaire, coin perdu (boutique, lodge) au milieu de la Namibie: tableau avec la pluviométrie des années récentes.

pressions violentes. A Swakopmund en Namibie, nous avons touché du doigt la réalité de ces quartiers avec un guide local (selon lequel il resterait aujourd'hui dangereux pour des touristes de s'y promener seuls). Le type de maisons construites dans trois townships adjacents différait selon le groupe tribal (maisons à une, deux ou trois pièces); ceci nous dit-on sans autre justification que d'attiser les tensions entre ces groupes, amenés à se jalouser à cause de telles différences injustifiables. Bien que l'apartheid ait été aboli avec succès, le caractère ethnique/racial de ces quartiers d'habitation ne devrait guère changer. Même les Noirs qui par hypothèse acquièrent une certaine prospérité ne chercheraient pas à habiter un quartier blanc où ils seraient isolés, mais restent dans le township, communauté de vie où ils sont nés, sont allés à l'école et à l'église, où la solidarité reste forte.

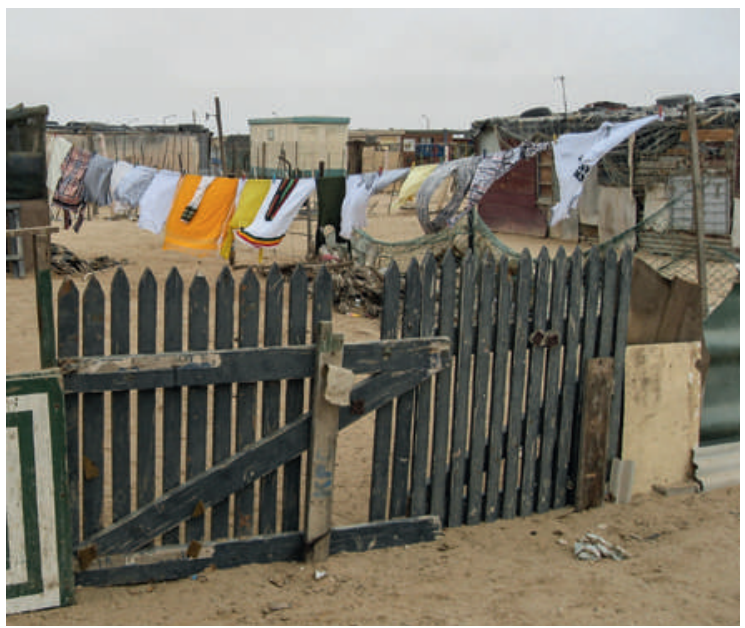

Dans le township de Mondesa, à Swakopmund, Namibie.
Héritage allemand de la Namibie. Elle a été une colonie allemande, grosso modo de 1880 à 1915 . Histoire marquée par de très mauvais traitements, notamment le génocide (à 80\%) du groupe des Hereros. Mais c'est un moteur du secteur économique majeur qu'est aujourd'hui le tourisme. A Swakopmund, sur la côte atlantique, on reste songeur devant les bâtiments de l'époque: résidence du gouverneur, tribunal, maisons de commerce et d'habitation; des églises dont une baroque qui vous transporte en Bavière. Nombreuses enseignes de Bäckerei-Konditorei. Le soir, les touristes allemands (et des Suisses) remplissent jusqu'au dernier siège de grands restaurants et retrouvent l'ambiance des brasseries de leur pays...

Dans les zones arides avec une activité d'élevage, on nous dit que plus de la moitié des fermiers sont allemands d'origine et parlent encore allemand (et afrikaans et anglais).

Géographie - Déserts. Dans cette très grande région, on trouve des chaînes de collines ou de montagnes, le plus souvent semi-désertiques. Il y a surtout de grandes étendues de pays plat, à perte de vue. Déserts de divers types, plus ou moins pierreux, plus ou moins sablonneux; avec un peu de végétation herbeuse ou arbustive. Parfois des zones d'immenses cônes de matériel d'origine volcanique aux belles couleurs des registres ocre, orange, mauve, violet. De loin en loin, prenant un chemin qui quitte la grand'route, on va trouver une ferme, loin... Rares agglomérations, quelques dizaines de maisons.

Des endroits particuliers qui valent le détour. Le Fish River Canyon, au sud de la Namibie, sur un affluent de la rivière Orange. Expérience comparable à celle qu'on fait au Grand Canyon d'Arizona: immensité plate et, tout d'un coup, énorme tranchée. Ici elle est très large $(27 \mathrm{~km})$, avec $550 \mathrm{~m}$ de profondeur seulement.

Il faut évoquer bien sûr le désert de sable du Namib qui a donné son nom au pays, sur la côte. Sur mille six cents kilomètres et une largeur de 50 à $160 \mathrm{~km}$. Très hautes dunes.

Les guides touristiques soulignent qu'il ne faut pas rater le coucher du soleil - ou son lever - à plusieurs de ces endroits... Des moments parfois magiques! Pour moi, cela a été le cas à nos campements de Bloedkoppe et de Spitzkoppe, au centre de la Namibie. Spitzkoppe est appelé le "Cervin namibien", sa silhouette - pyramide de granit rouge - fait effectivement penser à notre sommet national. J’y ai vécu une fin de journée exceptionnelle, dans un environnement de gros blocs polis évocateurs de formes de personnes ou d'animaux divers, changeant à mesure qu'on se déplace et que le jour décline. Beaux 


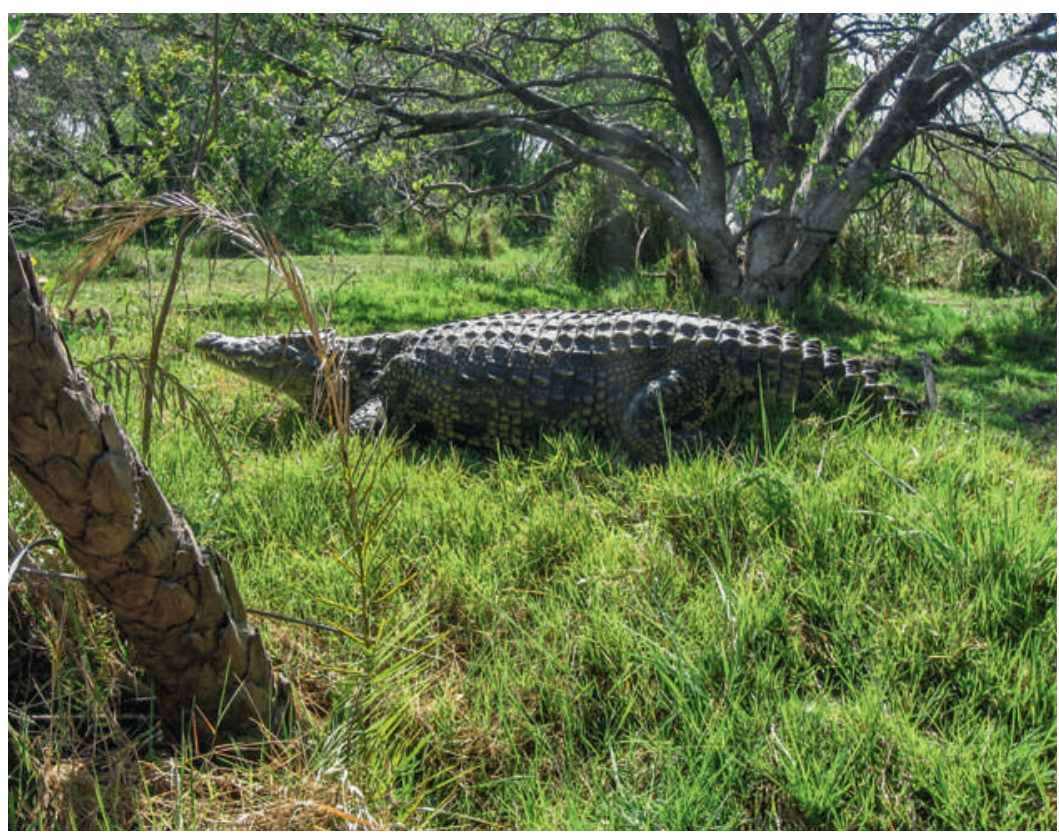

Dans le delta de l'Okavango (Botswana), où on se déplace en bateau. Beaucoup de crocodiles! oiseaux. Des familles de damans, cousins de nos marmottes. Ailleurs, une aire d'aigle accrochée haut dans une falaise, ici une trace de serpent ou de guépard; groupes d'antilopes diverses.

A ne pas négliger, le riche patrimoine de peintures rupestres de la Namibie. Quantité de sites, avec des dizaines de milliers de fresques témoins des sociétés d'éleveurs qui y ont vécu. On est frappé de la similitude (pour le non-spécialiste en tout cas) entre ces œuvres préhistoriques et celles qu'on connaît en Europe.

La faune. A Walvis Bay (Namibie), sur une grande plage, colonie d'otaries à perte de vue, en rangs serrés sur le sable ou jouant dans la mer. Vision incroyable il doit y avoir vraiment beaucoup de poissons pour en nourrir autant! Dans les parcs nationaux que nous avons vus (Etosha, Chobé), quantités d'animaux. Que ces bêtes sont impressionnantes (éléphants, rhinocéros, buffles, lions, un rare léopard), qu'elles sont belles (girafes, zèbres, quantités d'antilopes différentes dont le springbok, animal totem de l'Afrique du Sud, l'oryx, totem de la Namibie, le koudou, celui du Zimbabwe). Grands troupeaux, qui peuvent subitement quitter leur sérénité habituelle et se lancer dans une course panique à cause d'un danger effectif ou pressenti. Et des hyènes, chacals, des phacochères, des mangoustes. Beaucoup d'autruches, des outardes, des marabouts. Varans et lézards grands et petits. Crocodiles. Hippopotames , qu'on peut entendre gro- gner et manger, la nuit près du campement, dans le delta sans issue de l'Okavango au Botswana - où on voit aussi beaucoup d'aigles pêcheurs, magnifiques oiseaux blancs et noirs.

Chutes Victoria. Millions de mètres cubes d'eau tombant de $100 \mathrm{~m}$. dans une gorge profonde depuis un bord, une lèvre («rim») de plus d'un kilomètre et demi, alors qu'en amont le Zambèze se promène paresseusement dans un environnement plat. Marchés artisanaux, multiples propositions (vols en hélicoptère, rafting, saut à l'élastique...), résidences touristiques, des plus simples aux plus luxueuses. Et le Victoria Falls Bridge (route et chemin de fer) entre Zimbabwe et Zambie, qui aura bientôt cent ans.

Courrier. Surprise au retour: j'avais envoyé une vingtaine de cartes postales, de trois pays, en le faisant tôt de manière qu'elles arrivent avant moi. Or, très peu étaient chez leurs destinataires; la plupart ont nécessité près d'un mois. Dissonance, par rapport à ma bonne impression générale...

Pour finir, deux impressions particulières - vu les limites de mes observations. leur «solidité» ne peut être garantie:

Relations raciales. Il y a évidemment encore de grands différentiels de conditions socio-économiques, qui ne sont pas prêts de disparaître. Toutefois, dans ces pays qui ont connu la séparation stricte de l'apartheid (y compris interdiction des mariages et même de relations sexuelles interethniques), la coexistence paraît aujourd'hui se passer plutôt bien. Nous n'avons pas observé de manifestations d'arrogance ou de mépris; avons vu des Blancs et des Noirs collaborant dans la vie quotidienne dans le calme et la courtoisie. Bien sûr, cela c'est la vie "visible», dans la rue ou les commerces, il en va peut-être différemment dans d'autres circonstances.

Economie. Depuis quelques années on lit que le taux de croissance économique est fort dans la plupart des pays africains. Je suis à vrai dire méfiant de ces appréciations globalisantes, craignant qu'elles ne soient indûment optimistes (on se demande si quelques nababs amassant de grandes fortunes font monter les chiffres moyens alors que le "common man" reste pratiquement sans ressources et sans perspectives). Néanmoins, à plusieurs reprises et sans vraiment que j'y prenne garde, me souvenant de pays d'Afrique où j'ai vécu ou que j'ai visités, m'est venu à l'esprit que oui, il y a une élévation globale du niveau de vie et de ressources. Bonne nouvelle si cela est vérifié un peu largement. 